\title{
Noise decouples covariances from interaction strength
}

\author{
D Grytskyy ${ }^{1 *}$, T Tetzlaff $^{1}$, M Diesmann $^{1,2}$, M Helias $^{1}$ \\ From Twenty Second Annual Computational Neuroscience Meeting: CNS*2013 \\ Paris, France. 13-18 July 2013
}

Correlated neural activity is a known feature of the brain [2] and evidence increases that it is closely linked to information processing [1]. In our recent work we have shown how to map different network models, including binary networks, onto linear dynamics [4]. For binary neurons the mean-field approach takes random fluctuations into account to accurately predict the average activity in such networks [5]. Expressions for covariances follow from a master equation [3]. Binary neurons with a Heaviside gain function are inaccessible to the classical treatment [3]. Based on our earlier preliminary results [6] here we show how random fluctuations generated by the network effectively linearize the system of binary neurons, including the case of the Heaviside gain function, and how they implement a self-regulating mechanism which renders population-averaged covariances independent of the synaptic coupling strength. Figure 1A, B illustrate this invariance.

The mechanism is based on the increase of fluctuations in the input signal in proportion to the synaptic weight. The fluctuations cause portions of the gain function with smaller slope to be visited more frequently, effectively reducing the transmission gain. This keeps the linearized system away from instability, with the eigenvalues of its effective connectivity matrix bounded by a constant less than unity (see Figure 1C). Although of local origin the mechanism controls global features of the network dynamics.

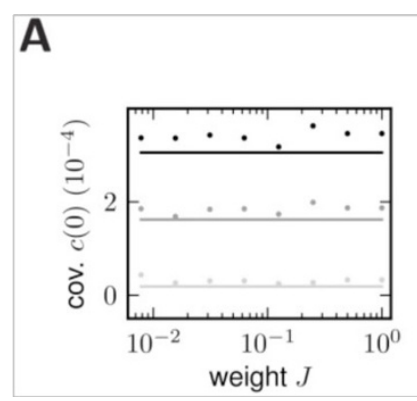

B

C
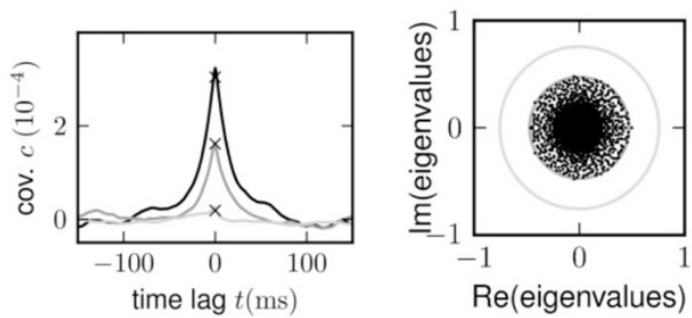

Figure 1 A Zero time-lag cross covariance averaged over pairs of excitatory (black) and inhibitory (light gray) cells and of one excitatory and one inhibitory neuron (gray) in simulation (dots) and theory (lines). B Cross covariance functions averaged as in A (same gray code) obtained from simulations at one coupling strength. Crosses show the analytical prediction. $\mathbf{C}$ Set of eigenvalues of a random connectivity matrix after linearization (black dots) with the corresponding spectral radius (gray circle) and the maximum radius for any synaptic strength (light gray circle).

\footnotetext{
* Correspondence: d.grytskyy@fz-juelich.de

${ }^{1}$ Inst. of Neuroscience and Medicine (INM-6) and Inst. for Advanced

Simulation (IAS-6), Jülich Research Centre and JARA, Jülich, Germany

Full list of author information is available at the end of the article
}

(c) 2013 Grytskyy et al; licensee BioMed Central Ltd. This is an Open Access article distributed under the terms of the Creative Commons 


\section{Acknowledgements}

We gratefully acknowledge funding by the Helmholtz Association: HASB and portfolio theme SMHB, the Next-Generation Supercomputer Project of MEXT, EU Grant 269921 (BrainScaleS). All network simulations were carried out with NEST (http://www.nest-initiative.org).

\section{Author details}

${ }^{1}$ Inst. of Neuroscience and Medicine (INM-6) and Inst. for Advanced Simulation (IAS-6), Jülich Research Centre and JARA, Jülich, Germany.

${ }^{2}$ Medical Faculty, RWTH Aachen, Aachen, Germany.

Published: 8 July 2013

\section{References}

1. Cohen MR, Kohn A: Measuring and interpreting neuronal correlations. Nat Rev Neurosci 2011, 14(7):811-819.

2. Gerstein GL, Perkel DH: Simultaneously recorded trains of action potentials: analysis and functional interpretation. Science 1969, 881(164):828-830.

3. Sompolinsky $\mathrm{H}$ : Theory of correlations in stochastic neural networks. Phys Rev E 1994, 50(4):3171-3191.

4. Grytskyy D, Tetzlaff T, Diesmann M, Helias M: Taming the model zoo: A unified view on correlations in recurrent networks. BMC Neurosci 2012 , 13(1):147.

5. van Vreeswijk CA, Sompolinsky H: Chaos in neuronal networks with balanced excitatory and inhibitory activity. Science 1996, 274:1724-1726.

6. Grytskyy D, Tetzlaff T, Helias M: Invariance of covariances arises out of noise. AIP Conf Proc 2013, , 1510: 258-262.

doi:10.1186/1471-2202-14-S1-P164

Cite this article as: Grytskyy et al:: Noise decouples covariances from interaction strength. BMC Neuroscience 2013 14(Suppl 1):P164.

\section{Submit your next manuscript to BioMed Central and take full advantage of:}

- Convenient online submission

- Thorough peer review

- No space constraints or color figure charges

- Immediate publication on acceptance

- Inclusion in PubMed, CAS, Scopus and Google Scholar

- Research which is freely available for redistribution

Submit your manuscript at www.biomedcentral.com/submit 